\title{
Multicellular Individuality: The Case of Bacteria
}

\author{
Rafael Ventura ${ }^{1,2}$ (1) \\ Received: 18 July 2018 / Accepted: 21 January 2019 / Published online: 25 February 2019 \\ c) Konrad Lorenz Institute for Evolution and Cognition Research 2019
}

\begin{abstract}
Recent attention to complex group-level behavior amongst bacteria has led some to conceive of multicellular clusters of bacteria as individuals. In this article, I assess these recent claims by first drawing a distinction between two concepts of individuality: physiological and evolutionary. I then survey cases that are representative of three different modes of growth: myxobacteria (surface-attached agglomerative growth), Bacillus subtilis (agglomerative growth not attached to a surface), and cyanobacteria (filamentous growth). A closer look at these cases indicates that multicellular individuality among bacteria is remarkably complex. Physiologically, the three cases of multicellular clusters do not form physiological individuals. But matters are different when it comes to evolutionary individuality; although multicellular clusters that grow by agglomeration are not highly individuated, filament-forming groups achieve a relatively high degree of individuality. I also suggest that debates about bacterial multicellular individuality may have been obscured by a failure to see that selection on highly individuated groups is by no means the only mechanism to bring about the complex group-level behaviors that have led some to view bacteria as multicellular individuals.
\end{abstract}

Keywords Bacteria $\cdot$ B. subtilis $\cdot$ Cyanobacteria $\cdot$ Individuality $\cdot$ Multicellularity $\cdot$ Myxobacteria

\section{Introduction}

Bacteria are often found in spatially cohesive clusters of cells-multicellular clusters of great architectural and social complexity in which intricate cell-to-cell communication and well-orchestrated group behavior take place (Claessen et al. 2014; Papenfort and Bassler 2016). Attention to phenomena of this sort has led some to question the view that bacteria are unicellular individuals (Shapiro 1988; Mendelson et al. 1997; Ereshefsky and Pedroso 2013, 2015). Their suggestion is that we should reevaluate our outlook on levels of organization among bacteria and instead think of multicellular clusters as individuals.

However, recent work on bacterial individuality has for the most part failed to attend to the high degree of variation across different bacterial groups. For example, Ereshefsky and Pedroso $(2013,2015)$ argue that some multi-species groups of bacteria are evolutionary individuals but make general statements about them with little or no qualification.

\footnotetext{
Rafael Ventura

rhtventura@gmail.com

Duke University, Durham, NC, USA

2 Bilkent University, Ankara, Turkey
}

Clarke (2016) opposes the view that bacterial multicellular clusters are evolutionary individuals but likewise overlooks that different bacterial groups differ in how individuated they are. Yet biological individuality comes in degrees. This means that we should shift the focus from whether multicellular clusters of bacteria are individuals to the question of how individuated they are. It also means that the question of bacterial multicellular individuality should be addressed on a case-by-case basis. ${ }^{1}$

To this purpose, I begin by emphasizing a distinction between two especially prominent concepts of individuality: a functional or physiological concept, and an evolutionary or Darwinian one (Pradeu 2010; Godfrey-Smith 2013). According to the physiological concept, biological individuals display a high degree of functional cohesion and integration among component parts; according to the evolutionary concept, individuals are members of a population capable of undergoing evolution by natural selection. Once this distinction is drawn, I propose different individuating mechanisms and delimitation criteria to help us

\footnotetext{
${ }^{1}$ Skillings (2016) also considers a specific case of putative multicellular individuality, but his focus is on symbiotic associations between multispecies communities of microorganisms and marine invertebrates - not on the sort of monospecies bacterial groups that I consider here.
} 
operationalize these concepts. In the case of evolutionary individuality, I take the main individuating mechanism to be a narrow bottleneck between generations. In the case of physiological individuality, it is the division of metabolic labor among terminally differentiated cells that acts as an individuating mechanism. The extent to which generations go through a narrow bottleneck and the number of terminally differentiated cell types can therefore serve as delimitation criteria in these two cases. After operationalizing individuality concepts in this way, I address the question of bacterial multicellular individuality by bisecting it into two questions: in an evolutionary sense, how individuated are multicellular clusters of bacteria? And how individuated are they in a physiological sense?

I then address these questions by surveying three monospecies groups of bacteria: myxobacteria, Bacillus subtilis, and cyanobacteria. I focus on these three cases for two reasons. First, they display the type of complex group-level behavior that has led some to tout bacteria as multicellular individuals. Second, these three groups of bacteria are representative of three distinct types of multicellular growth: surface-attached agglomerative growth (e.g., myxobacterial fruiting bodies), agglomerative growth not attached to a surface (e.g., macrofiber and pellicle formation in Bacillus subtilis), and filamentous growth (e.g., cyanobacterial filaments).

Several other multicellular clusters of bacteria display similar growth patterns. In cystic fibrosis patients, for example, Pseudomonas aeruginosa colonies attach to surfaces of the respiratory tract (Winstanley et al. 2016). Another example is the symplasmata of Pantoea agglomerans-multicellular colonies protected by a polysaccharide capsule that are often found attached to plant roots and leaves (Tecon and Leveau 2016). In liquid medium, Acinetobacter spp. and other bacteria that grow by agglomeration also form pellicles at the liquid-air interface (Martí et al. 2011). And several species of Streptomyces exhibit complex filamentous growth in solid medium (Jones and Elliot 2018). Given that many different bacterial taxa follow one of these three growth patterns, conclusions about the three case studies presented here can therefore be generalized to other taxa.

As I show in this article, the picture that emerges from a closer look at these three bacterial cases is that bacterial multicellular individuality is remarkably complex. Myxobacteria and Bacillus subtilis, for one, display a very low degree of evolutionary individuality at the multicellular level. This is because multicellular clusters of bacteria that grow by agglomeration do not undergo a narrow bottleneck. But this is not the case of cyanobacteria. Filament-forming bacteria undergo a single-celled bottleneck every time a filament detaches from the larger colony. In a physiological sense, on the other hand, myxobacteria, Bacillus subtilis, and cyanobacteria do not form highly individuated multicellular clusters. Although there is some division of metabolic labor among terminally differentiated cells at a local level, the division does not take place at the group level. Regardless of growth pattern, these multicellular clusters of bacteria therefore do not display a high degree of physiological individuality at the multicellular level.

The article proceeds as follows. The second section describes two different concepts of biological individuality, together with their individuating mechanisms. The third section provides case studies of three commonly discussed groups of bacteria. I then argue in the next section that much of the debate about bacterial individuality has been in part obscured by a failure to differentiate between distinct mechanisms that permit complex social behaviors to flourish. If complex behavior at the group level can also arise by natural selection at a lower level, mechanisms other than selection on highly individuated groups might be able to explain the persistence of the complex group-level behaviors that permeate bacterial organization.

\section{Two Concepts of Individuality}

A good deal of the current discussion about bacterial individuality originated with Shapiro's (1988) suggestion that bacteria can form tight associations of cells that are analogous to multicellular organisms. Drawing on cases of bacterial cooperation, such as cellular differentiation, fruiting body formation, and social motility, Shapiro proposed that "in many ways an individual bacterium is more analogous to a component cell of a multicellular organism than it is to a free-living, autonomous organism" (p. 82). In his article, the emphasis was on the coordinated behavior and integrated function of bacterial communities. Examples of metabolic specialization in cyanobacterial filaments, swarming in Proteus mirabilis colonies, communal feeding in myxobacteria, and other "sophisticated events specific to multicellularity" (p. 83) served to substantiate Shapiro's claim that bacteria behave and grow much like other cases of multicellular organisms, such as metazoans (Buss 1987).

It is not always clear whether Shapiro (1988) regards bacteria as multicellular individuals, as the title of his article seems to suggest, or if he simply subscribes to the less substantial claim that bacteria display complex group-level behavior. Regardless of Shapiro's initial intentions, some now embrace the view that individuality is a trait of multicellular clusters of bacteria. For example, Ereshefsky and Pedroso $(2013,2015)$ claim that we should think of multispecies groups of bacteria as multicellular individuals. These authors agree that multicellular clusters of bacteria are not evolutionary individuals in the sense that they lack the reproductive mechanisms usually associated with such individuals. But they argue that we should accept a more 
inclusive notion of individuality according to which multicellular clusters of bacteria are also individuals. An earlier expression of this trend is Mendelson et al. (1997), for whom multicellular individuality among prokaryotes "implies cooperativity and the shifting of selection from operating at the level of individual cells to that of the multicellular entity" (p. 339).

Pronouncements like this suggest that there may be some confusion in the debate over bacterial individuality. Initially, attention to multicellular phenomena was aimed at doing justice to the behavioral and physiological complexities of bacterial groups; more recently, the significance of multicellular aggregates as evolutionary individuals has also been contemplated. Although some oppose the view that multicellular clusters of bacteria should be considered evolutionary individuals (Nadell et al. 2009; Clarke 2016), there has been a clear shift in the debate: whereas the focus was originally on group-level functional integration, now the debate is cast in terms of group selection and evolutionary individuality at the multicellular level.

How, then, should we think of multicellular clusters of bacteria? Although the difficulty in tackling this question is partly due to the intricacies inherent to bacterial forms of life (microscopic bacteria are not the kind of organisms our concepts were designed to grapple with), the impasse can be alleviated by distinguishing two different notions of biological individuality: a functional or physiological concept, and an evolutionary or Darwinian one (Pradeu 2010; GodfreySmith 2013). Although other individuality concepts have also been proposed-e.g., ecological individuality (Huneman 2014; Pradeu 2016) - I focus here on physiological and evolutionary concepts because they have received the most attention recently, especially in debates about bacterial multicellular individuality.

According to the physiological concept, biological individuals are homeostatic groups of entities that display a high degree of functional integration among component parts-a view that goes at least as far back as Huxley (1912). On this view, the main feature of an individual is thus that it is "a regulated unit that persists through time" (Pradeu 2016, p. 800). According to the evolutionary concept, on the other hand, individuals are the units of selection. Slightly different criteria have been proposed for what counts as an individual in this sense. But common to all of them is the assumption that Darwinian individuals inherit an amalgam of traits (heritability) that vary within a population (variation) and that potentially contribute to their differential rates of survival and reproduction (differential fitness) (Lewontin 1970)

At this point, a brief digression into the species concept will prove illuminating. In debates about species, it is now common to distinguish between species concepts, methods of species delimitation, and processes or mechanisms of speciation (de Queiroz 1998, 2007). A species concept gives a definition that specifies what properties must be present for a group of organisms to count as a species. A method of species delimitation offers reliable criteria for the identification of species. And a process of speciation is a mechanism for bringing about the properties that species concepts pick out-e.g., sympatry or allopatry.

Attention to these distinctions has helped move forward the debate about species by dissolving merely verbal disputes and locating sources of real disagreement. Similar distinctions can and should be made in the debate about biological individuality as well. I therefore propose a tripartite division of questions about biological individuality into issues pertaining to different individuality concepts, methods or criteria for the delimitation of individuals, and individuating mechanisms. A concept of individuality specifies properties that must be present for a group of organisms, cells, or subcellular structures to count as an individual. A method for the delimitation of individuals offers criteria for the identification of individual boundaries. And a process of individuation is a mechanism by which groups of entities become individuated.

The evolutionary and physiological concepts of individuality given above specify what properties must be present for a group of entities to count as an evolutionary or a physiological individual. As already indicated, these are therefore two different concepts. In the case of evolutionary individuality, the concept specifies that what counts as an individual is a member of a population capable of evolving by natural selection. In the case of physiological individuality, the concept specifies that an individual is a metabolically cohesive whole.

But both concepts do not specify methods of delimitation or mechanisms of individuation. What could these be? Godfrey-Smith (2009) gives an especially detailed and much-debated account of individuating mechanisms in the case of evolutionary individuals. His starting point is the observation that one of the central characteristics of Darwinian individuals is the ability to "de-Darwinize" entities at a lower level (Godfrey-Smith 2009, p. 125): individuals at the higher level of organization suppress the workings of natural selection at the lower level, at the same time that evolutionary processes at the higher level proceed unchecked. A powerful mechanism to achieve this is a bottleneck between generations (Roze and Michod 2001). A bottleneck reduces genetic variation and fitness dependence on intrinsic character at the lower level. With little variation and reduced dependence on intrinsic character (which tends to be heritable), the potential for evolution between lower-level particles is restrained. There is thus impairment of two elements in the triad of variation, heritability, and differential fitness that define a Darwinian individual as a member of an evolving population. 
In the case of physiological individuality, an efficient mechanism to reinforce individual boundaries is the division of metabolic labor among terminally differentiated parts (Pradeu 2016). When dealing with groups of cells, individual boundaries are therefore maintained by the division of metabolic labor amongst terminally differentiated cells. Terminal differentiation makes different cells mutually dependent on one another, ensuring that the group of cells is functionally cohesive and integrated. In microbiology, attention to this type of mechanism can be traced back to Shapiro's (1988) initial assertion that bacteria should be understood as multicellular organisms.

The distinction between these individuating mechanisms also gives us clear methods of delimitation-i.e., readily distinguishable criteria for the identification of individuals. Given the role of an intergenerational bottleneck as a mechanism of evolutionary individuation, the corresponding criterion for the identification of evolutionary individuals is the extent to which generations undergo a bottleneck: the narrower the bottleneck, the more individuated the collection of cells in an evolutionary sense. To determine how individuated groups of bacterial cells are in an evolutionarily sense, we must therefore determine the degree of intergenerational bottleneck in the group of cells. This means that, in an evolutionary sense, highly individuated multicellular clusters of bacteria should be expected to undergo a narrow bottleneck at some point during their life cycle.

In the case of physiological individuality, the main mechanism of individuation is the division of metabolic labor amongst terminally differentiated cells. The corresponding criterion for the identification of physiological individuals is therefore the degree to which metabolic labor is divided amongst terminally differentiated cells: the greater the division of metabolic labor amongst terminally differentiated cells, the more individuated the collection of cells in a physiological sense. To determine how physiologically individuated groups of bacterial cells are, we must therefore determine the number of terminally differentiated cell types. This means that, in a physiological sense, highly individuated multicellular clusters of bacteria should be expected to harbor a large number of terminally differentiated cell types.

Bearing in mind these distinctions between individuality concepts, individuating mechanisms, and criteria of individuation, in the next section I investigate how individuated some prominent groups of social bacteria actually are. These distinctions will permit me to address the question of bacterial multicellular individuality by breaking it down into questions that are easier to answer. First, how individuated are multicellular clusters of bacteria in an evolutionary sense? To answer this question, I determine the extent to which multicellular clusters of bacteria undergo a narrow bottleneck between generations. Second, how individuated are multicellular clusters of bacteria in a physiological sense? To answer this question, I determine the extent to which multicellular clusters of bacteria are composed of terminally differentiated cells.

\section{Social Bacteria}

As will become clear in what follows, the behavior of bacterial multicellular clusters can be surprisingly complex and multifarious. The three case descriptions that follow are therefore inherently simplified. But I nonetheless hope to extract considerations from them that can be usefully generalized.

\section{Myxococcus xanthus}

Myxobacteria have often been advertised as a case of multicellular individuality. Alternating between vegetative growth and a developmental cycle that culminates in sporulation, the myxobacterium Myxococcus xanthus is a much-studied case. In the presence of a solid surface to provide support for motility, individual cells respond to a nutritional downshift by releasing chemoattractants, thereby inducing agglomeration (Munoz-Dorado et al. 2016). Although little is known about the identity of the chemicals involved, cell density seems to play a decisive role in activating developmental genes: scarcity in amino acids (their preferred source of nutrients) downregulates the expression of growth-related genes and promotes activation of a population-sensing pathway, both of which are essential steps in the multistage process of fruiting body formation (Shimkets 2000). For unknown reasons, only about $15 \%$ of all the cells partaking in the developmental cycle successfully enter dormancy and are able to sporulate, while the remaining portion of the population undergo autolysis and die (Shimkets et al. 2006). Even after spore germination, $M$. xanthus's social configuration can remain stable throughout most of their vegetative growth phase: it has long been known that a large number of cells are able to coordinate their activity as parts of a multicellular body in order to entrap and digest prey organisms (Burnham et al. 1981).

This brief characterization of the $M$. xanthus life cycle suffices to address some of the major themes in multicellular individuality among myxobacteria. In fact, fruiting body formation and communal feeding is what first led Shapiro (1988) to observe that "many Myxobacteria never exist as single cells" (p. 83). But does this give us a good reason to conclude that $M$. xanthus and other related species are, in a physiological sense, multicellular individuals? Research indicates that high cell density correlates with more robust growth rates in stressful environments, suggesting that at least under certain conditions a communal lifestyle alters gene expression and metabolic activity of the individual 
cells involved (Velicer et al. 2008). Similarly, fruiting bodies may serve to preserve high cell density throughout the dormant period, which facilitates growth once cells break the dormancy (Velicer et al. 2008). There is thus compelling evidence for the view that social behavior can be highly organized among individual cells.

However, this alone does not justify the claim that myxobacterial aggregates are physiological individuals. For one, there is no metabolic division of labor amongst terminally differentiated cells within $M$. xanthus colonies. This is important because according to the model proposed by Boyle and Lenton (2006) metabolic division of labor among differentiated cells is the most significant factor to explain the cohesion of a multicellular cluster. To support this model, they first assume spatiotemporal fluctuations in the availability of environmental resources-a very likely scenario in natural habitats. Given this assumption, metabolic robustness resulting from "a group-level physiology that is able to 'buffer' the fluctuation that individuals within a group experience relative to autonomous individuals" (p. 833) will then maintain the group's cohesion. That is, metabolic division of labor amongst specialized cell types imbues the group with greater metabolic versatility. This in turn fortifies the physiological ties among different parts, which aggregate into a mutually dependent cluster of cells. In a physiological sense, therefore, multicellular individuals arise when terminally differentiated cells split metabolic tasks. When this occurs, the collection of mutually dependent cells becomes a metabolically autonomous whole. If the characterization of myxobacterial lifecycle provided above approximates the real sequence of events in nature, division of metabolic labor among terminally differentiated cells plays no significant role in myxobacterial communities.

Similar worries arise with regard to an evolutionary understanding of myxobacterial individuality. Colonies of $M$. xanthus are founded by clumps of many spores that tightly adhere to one another (Shimkets et al. 2006). Thus, multicellular clusters of $M$. xanthus do not undergo a narrow bottleneck at any point of their life cycle, and variation among cells can persist. That individual cells within a myxobacterial population usually harbor a substantial reservoir of heritable variation, at least in a potential state, is further suggested by the rapid evolution of developmental cheating and cooperative behavior regulated by novel mutations (Velicer et al. 2000; Velicer and Yu 2003).

\section{Bacillus subtilis}

Bacillus subtilis exhibits a wide range of collective behaviors (Vlamakis et al. 2013). I focus on two that are particularly relevant for questions regarding bacterial individuality: macrofiber and pellicle formation, and endosporulation. In nutrient-rich media, macrofibers form when growth is rapid and cells fail to detach after binary fission: as cells expand, the resulting filament elongates, twists, and coils into a spherical mass of intertwined fibers (Mendelson et al. 2002). The structure begins to decay as soon as the macrofiber ball reaches a critical point in its development. Shedding fragments then give rise to progeny fibers in the same culture as the parental macrofiber-a process apparently related to "the breakdown of multicellular structure caused by the completion of cell separation" (Mendelson 1999, p. 471). B. subtilis can also form pellicles-a multicellular mat of cells at the liquid-air interface (Steinberg et al. 2018). Although focus is here on multicellular aggregates that grow in liquid medium, high-density populations of $B$. subtilis can also propagate as surface-attached colonies of branched filaments, which acquire complex and diverse morphologies with regular patterns of circumferential and sectoral differentiation. In both liquid and solid media, once nutrients have been depleted after the exponential growth phase, extra- and intracellular signals then combine to induce endosporulation (Sonenshein 2000). During this process, cells first undergo asymmetrical division, giving rise to a mother cell that completely engulfs the forespore compartment. The mother cell eventually lyses and the mature, stress-resistant endospore is released.

Phenomena of this sort have prompted some to view populations of $B$. subtilis as a multicellular individual. An example is Branda and Kolter (2004), who understand endosporulation as a case of division of labor in which the mother cell specializes in metabolic activities complementary to the spore's reproductive function. In arguing for this view, they observe that "cells committed to spore formation cannot grow vegetatively, and the mother cells that nurture the developing spores are terminally differentiated in the sense that they eventually lyse to release the spore" (p. 26). It is true that a certain degree of metabolic division of labor takes place locally, but I do not think that we would be justified in making use of the physiological concept of individuality at the group level. As with fruiting body formation by $M$. xanthus, the community of $B$. subtilis cells as a whole is-in a physiological sense-poorly integrated. Although the crossing of a cell-density threshold seems to trigger endosporulation (Kroos et al. 2008), the physiological events that follow do not depend on different parts of the colony. That is, splitting the filamentous mass of bacterial cells into smaller ones would not affect endospore formation, as long as the appropriate cell density is preserved. So when it comes to endospore formation, the different parts of the whole are not mutually dependent. Nor is complex colony morphology in solid medium necessarily an indication of division of labor. If we can transpose insights gained from experiments with Escherichia coli to the case of B. subtilis, 
rigid patterns of concentric rings may stem from self-generated chemical fields in the substrate-not from genetically controlled, terminal cell differentiation. ${ }^{2}$

Corroborating the view that clusters of B. subtilis do not form cohesive multicellular individuals in a physiological sense is the case of macrofiber and pellicle formation. More dramatically in these cases, cells within macrofibers and pellicles do not manifest any degree of terminal differentiation at all. On account of the absent division of metabolic labor amongst terminally differentiated cells, Mendelson et al. (1997) conclude that "morphogenesis of higher organisms differs from that found thus far in macrofibers" (p. 353). Hence, in spite of physical contiguity among cells, there is little physiological integration or cohesion among them.

What about evolutionary individuality: do multicellular aggregates of $B$. subtilis form Darwinian individuals? Prima facie, certain properties of macrofibers may encourage us to think so. By shedding fragments that can give rise to offspring, macrofibers seem able to form discrete lineages. ${ }^{3}$ But suggestive of the marginal degree of individuality at the multicellular level is the fact that mature macrofibers give rise to a new generation of macrofibers by disintegrating into fragments of different sizes (Mendelson et al. 1997). That is to say, there is no narrow bottleneck to purge deleterious mutations, or to minimize potentially conflictive genetic heterogeneity (Grosberg and Strathmann 1998). Because of this, it would not be appropriate to talk of evolutionary individuality at the multicellular level in this case. ${ }^{4}$

Likewise, there are no compelling reasons to regard spore or pellicle formation as a means of creating a narrow bottleneck between colony generations. Genes responsible for endosporulation are regulated by cell density signals (Burkholder and Grossman 2000). In response to a dearth of nutrients and other environmental cues, cells export factors that reflect the population density. Cells will only sporulate

\footnotetext{
2 The formation of concentric rings seems to be dictated by environmental factors. Inoculating a new $E$. coli population next to an established colony causes the young colony to bypass "some of the stages of pattern formation to come into register with the older colony" (Shapiro 1997, p. 35). More than a temporal ontogenetic program, it is spatial location that regulates phenotypic differentiation in this case-a feature that motivates Andrews (1998) to conclude: "bacteria affect and are in turn affected by their environment much as are modular organisms" (p. 112).

3 This is one of the requirements in the transition to multicellularity envisioned by Okasha (2005): the requirement "that the collectives be sufficiently discrete to form identifiable ancestor-descendent lineages" (p. 1023).

${ }^{4}$ Again, if comparisons to colonies of E. coli are legitimate, sector differentiation is a good example of potentially conflictive genetic heterogeneity. Sectors are formed when genetic changes alter the functioning of a bacterium and consequently of its descendants. In some cases, differential reproductive output among sectors creates visible distortions around a colony's edge (Shapiro 1997).
}

if these factors cross a threshold concentration. This means that colonies of $B$. subtilis have found a way of sporulating in synchrony. When spores germinate, they are likely to do so simultaneously too, since small soluble molecules that diffuse in the medium trigger germination (Paidhungat and Setlow 2002). Given that spores are highly resistant and immotile entities, large numbers of spores will give rise to a new colony together, provided conditions are favorable for germination. Similarly, pellicle formation is initiated by cell clusters of variable sizes (Vlamakis et al. 2013). There is thus no dedicated mechanism for forcing pellicles to go through a narrow bottleneck between generations. For these reasons, endosporulation and pellicle formation do not seem able to efficiently reduce variation within multicellular aggregates: Darwinian processes at the cellular level can go on more or less unchecked.

\section{Heterocyst-Forming Cyanobacteria}

Cyanobacteria are photosynthetic prokaryotes, some of which grow filamentous structures in which multiple cells adhere together. In some cases, these filamentous structures are also capable of differentiating into a variety of cell types. One of the most remarkable examples of this is heterocyst formation. In the absence of combined nitrogen, some cells take up the ability to fix atmospheric nitrogen $\left(\mathrm{N}_{2}\right)$ into chemically useful forms. Heterocysts, as these cells are called, thus cover their own demand for nitrogen, as well as that of neighboring cells. But because nitrogenase-the nitrogen-fixing enzyme involved in this process-is highly sensitive to oxygen, heterocysts undergo complex regulatory and morphological changes, which isolate them from the poisonous presence of oxygen in the environment. These changes include loss of the ability to photosynthesize, making them dependent on the photosynthetic products of adjacent cells (Kumar et al. 2010). Some heterocystous cyanobacteria can also differentiate into short, motile filaments called hormogonia. In contrast to vegetative filaments, a hormogonium is usually wrapped in a hydrophilic envelope and may contain gas vesicles (Flores and Herrero 2010). Additionally, some cyanobacterial cells are able to turn into akinetes-dormant and highly stress-resistant spore-like cells.

Given that akinetes so closely resemble the spores of other social bacteria, I avoid dealing with them separately in the discussion that follows. Instead, I focus on heterocysts and hormogonia. Heterocystous filaments may at first look like promising candidates for multicellular individuals in a physiological sense. Schirrmeister et al. (2011), for one, seem to take the evolutionary advent of filamentous growth in both heterocyst-forming and non-heterocyst-forming cyanobacteria to have resulted "in changes in the organizational confines of the individual" (p. 12). Indeed, in the 
case of heterocystous filaments, there is both physiological heterogeneity and mutual dependence among parts: photosynthesizing cells and nitrogen-fixing heterocysts perform metabolic activities that are complementary and in nitrogenpoor environments can only thrive in tandem. So one could be tempted to see this as a genuine instance of group-level physiology, which acts as a "buffer" against environmental fluctuations. And to some extent, there are good reasons to think so. Interchange of metabolic products indeed confers functional integration to filaments interspersed with heterocysts and carbon-fixing cells. Even though the availability of nitrogen or carbon in the environment may oscillate, associations between nitrogen- and carbon-fixing cells result in metabolic robustness. This suggests some, albeit low, degree of physiological individuality.

But what are the members of this association and what is the physiological individual formed in this case? Each heterocyst-forming cyanobacterium has a characteristic, nonrandom pattern of heterocyst spacing (Kumar et al. 2010). Unsurprisingly, heterocyst location seems to correlate with the cells' ability to distribute fixed nitrogen along the filament. This accounts for the iterative pattern of filamentous subunits, each containing heterocysts in intercalary or terminal positions (or both, depending on the species). Thus, if nitrogen-fixing and carbon-fixing cells form any kind at all of multicellular associations with a high degree of functional integration, it seems more accurate to describe each subunit in which they occur-and not the whole mass of branching filaments-as a rudimentary form of physiological individual. This is because long filaments can split and shed fragments with no adverse consequences for the whole structure, whereas iterative modules of heterocysts and carbon-fixing cells cannot be easily divorced without loss of function. ${ }^{5}$

What about evolutionary individuality? Are there good reasons to regard multicellular clusters of cyanobacteria as evolutionary individuals? That is, do they go through a narrow bottleneck? In a sense, a hormogonium can be said to function as a narrow bottleneck between generations, even if the means by which this happens are quite disorienting at first. During hormogonia formation, the total number of cells increases, but DNA synthesis freezes during the whole process. This is possible because cells of filamentous cyanobacteria contain multiple genome copies, so hormogonia can develop through synchronized cell division in the absence of DNA replication (Adams 2000). As a result, the amount of genetic material remains constant, as it is partitioned into

\footnotetext{
${ }^{5}$ In some species of the genus Cylindrospermum, fragmentation may be an integral part of the process leading to regular heterocyst spacing: as cells distant from functional heterocysts become nitrogen-depleted and die, filamentous fragments multiply "with heterocyst formation then ensuing at both of the resultant ends" (Wolk 2000, p. 87)
}

smaller chunks and the cells multiply. Combining the proliferation of cell compartments with lack of DNA replication, a hormogonium reduces the amount of DNA per cell, but not the total number of genomes. Once the hormogonium resumes vegetative growth, cells and the genomes contained in them can nevertheless proliferate. Eventually they may fragment and differentiate into new hormogonia, completing the cycle. At the subcellular level, then, hormogonia certainly undergo a bottleneck: during hormogonia formation, the population of cells expands but the number of genome copies per cell decreases in comparison to the amount of DNA per cell during the vegetative growth phase. But as the bottleneck takes place at the cell level, hormogonia formation contributes to the individuation of cells-not of multicellular clusters.

However, the filamentous growth pattern of cyanobacteria does create a bottleneck at the level of multicellular clusters. This is because every time a filament fragments, all the cells that remain linked together are the descendants of a single cell. Cyanobacterial filaments are therefore largely uniclonal, as the whole colony undergoes a unicellular bottleneck every time it divides into daughter colonies. Perhaps the reason why this is not immediately obvious is that in most familiar life forms - and that usually means macroscopic creatures that resemble us-a unicellular bottleneck comes before the rapid multiplication of cells that ensues during ontogenesis. In the case of cyanobacterial filaments, on the other hand, cells multiply before undergoing a narrow bottleneck. It is thus only after cellular proliferation that the multicellular cluster undergoes a single-cell bottleneck. The process is not unlike the way in which experimentally evolved multicellular clusters of yeast undergo a unicellular bottleneck: in snowflake yeast, cells divide and remain serially attached to each other until short uniclonal branches detach from the mother colony (Ratcliff et al. 2012).

\section{Bacteria as Social Individuals}

Two general themes emerge from the cases examined above. First, multicellular aggregates of myxobacteria, B. subtilis, and cyanobacteria tend to exhibit a very low degree of physiological individuality. These multicellular clusters of bacteria are internally homogeneous and there is no or little division of metabolic labor amongst terminally differentiated cells. Even in the case of heterocyst-forming cyanobacteria, in which mutually dependent nitrogen-fixing and carbon-fixing cells metabolize in tandem, the notion of a semiautonomous module is highly idealized and most cells may exist as members of loosely connected filaments. In fact, detachment from the colony might even be an integral part of a bacterium's life cycle (Stoodley et al. 2002) and in 
some cases regulated by proteases that digest the extracellular matrix (Lee et al. 1996).

Second, most multicellular clusters of bacteria exhibit an equally low degree of evolutionary individuality. Multicellular clusters of bacteria that grow by agglomeration-be it attached to a surface (e.g., myxobacteria), or suspended in liquid medium (e.g., B. subtilis)—lack a narrow bottleneck that marks the transition between generations. This impedes the emergence of Darwinian individuals at the multicellular level. However, the case of filamentous cyanobacteria and other filament-forming bacteria is importantly different. As filaments are composed of a single cell layer, their growth pattern forces the multicellular cluster of cells down a single-cell bottleneck whenever the colony is disrupted and a filament is separated. This reduces the variation at the cell level, at the same time increasing the variation at the multicellular level. As a result, it is easier for Darwinian processes to act and individual boundaries to emerge at the multicellular level.

One obvious conclusion to draw from these considerations is that multicellular clusters of bacteria are not all alike. Some may exhibit a higher degree of individuality than others, so that there is not always a multicellular individual where there is a group of cells. That is, cells can interact as parts of a group to different degrees of evolutionary and physiological cohesiveness. Following this line of reasoning, multicellular clusters of bacteria could be understood as loose aggregates that stand between the two poles of a spectrum from minimal to maximal degrees of individuality, with some groups falling closer to the extremes than others.

But at this point one could still wonder: how is it possible, then, for some groups of bacteria to display all the features of complex social interactions that they do and still not qualify as full-fledged multicellular individuals? An answer to this question can be given by first thinking of prominent cases of bacterial multicellular behavior as involving the creation of a public good. Indeed, it is now standard to regard the production of extracellular matrix, stalk formation, and the differentiation into nitrogen-fixing cells and hormogonia as social behaviors that involve the creation of a public good (Crespi 2001; Cao et al. 2015; Dragoš et al. 2017). This is because they all result in the creation of "products that are costly for the individual to produce but that provide a benefit to the individuals in the local group or population" (West et al. 2006, p. 598). Some complex group-level behaviors among bacteria are thus legitimate examples of behaviors that produce public goods, because it would be advantageous for an individual bacterium to reap the benefits of their expression without investing in their production.

A good experimental treatment of such traits has been given to the phenomenon known as swarming in the social myxobacterium Myxococcus xanthus (Velicer and Yu 2003). Under certain conditions, swarmers move faster and farther than non-swarmers. But swarming also requires the production of an extracellular fibril that binds cells together and permits them to coordinate their movement. By knocking out the gene encoding this extracellular fibril, Velicer and $\mathrm{Yu}$ (2003) created strains that were defective in their swarming ability. They then subjected these mutant strains to a selective regime in which the outermost portions of the $M$. xanthus colonies were culled and allowed to infect new medium. Exposure to this selective regime eventually led to the appearance of strains that were able to swarm more efficiently than their mutant defective ancestors. Because the restored swarming ability seemed to depend on the presence of the extracellular fibril, but not on the same genes as in the wild type, Velicer and Yu (2003) concluded this was the evolution of a novel social behavior. Moreover, fibril production proved to slow down the growth rate of all swarming strains relative to non-swarming strains. This, then, seems to be a genuine case of a public good: costly for the individual to produce in isolation, but beneficial if there is selection for individuals to swarm cooperatively.

The fact that cooperative swarming evolved in Velicer and Yu's (2003) experiment indicates that selection for the production of a public good was able to prevail over selection against it. By analogy, production of other public goods in social bacteria, such as fruiting body formation, macrofibers, and heterocyst formation, suggests that selection for the production of these goods is generally stronger than selection against it. More importantly, if complex group-level behaviors of the type that led some to think of bacterial multicellular clusters as individuals can indeed be understood as a form of public good production, then it may be possible to explain their emergence and persistence without invoking selection on highly individuated groups.

This is because different individual-level mechanisms that do not require selection on highly individuated groups can favor the production of public goods (Travisano and Velicer 2004). By making the production of a public good dependent on the concentration of signaling molecules, as for example in the case of quorum-sensing mechanisms, bacteria have found a way to couple the production of a public good to a critical concentration of public good producers in the population. Policing mechanisms to punish free riders and mutual recognition are other forms of enhancing interaction among public good producers. Yet another way of achieving similar results is to structure the population in such a way that like correlates with like, and the multicellular clusters of bacteria considered here are a remarkable example of this. Filamentous growth prevents cells from interacting randomly; during aggregative growth, bacteria are likewise cemented to the extracellular matrix and thus forced to interact locally (Davies 2000). A population model that approximately captures this spatial configuration is that of a two-dimensional lattice divided into neighborhoods, in which each cell affects 
the fitness of its immediate neighbors (Godfrey-Smith 2008). In cases like this, it is simply population structure that accounts for the persistence of public goods.

Failure to recognize that selection at lower levels of organization may account for the emergence and persistence of complex group behavior that involves the production of a public good has possibly been the reason why multicellular clusters of bacteria have sometimes been confounded with evolutionary individuals. But this also means that there is no reason to think of multicellular clusters of bacteria that display complex group-level behavior as necessarily involving multicellular individuals.

\section{Conclusion}

In this article, I assess recent claims that bacteria should be understood as multicellular individuals. My discussion suggests that the debate on bacterial multicellular individuality has been obscured by a number of factors. First, failure to distinguish between two distinct concepts of individualitya physiological concept and an evolutionary one-has led some to conflate different questions and thus muddied the debate. A related point is that general debates about biological individuality, and in particular about bacterial individuality, have often failed to tease apart questions about individuality concepts from issues about individuating mechanisms and criteria of individuation. As I show in this article, attention to these distinctions can help advance the debate about bacterial multicellular individuality.

Second, recent work on bacterial individuality seems to proceed on the assumption that all bacteria are created equal. However, there is a large amount of variation among multicellular clusters of bacteria as to how individuated they are. Debates about bacterial multicellular individuality should therefore proceed not only with an eye towards the distinctions just mentioned, but also on a case-by-case basis. In a physiological sense, the three different cases considered here-surface-attached agglomerative growth (e.g., myxobacteria), agglomerative growth not attached to a surface (e.g., B. subtilis), and filamentous growth (e.g., cyanobacteria) - are not highly individuated. But matters are different in an evolutionary sense. Filamentous bacteria form highly individuated multicellular clusters, whereas bacteria that grow by aggregation do not.

A final point concerns the hierarchical structure of evolutionary processes. Many who now see bacteria as multicellular individuals appeal to complex group-level behaviors in defending their view. The reasoning seems to be that complex group-level behaviors could only evolve if selection were to act on highly individuated groups. But selection on highly individuated groups is by no means the only mechanism to bring about these traits. Mechanisms such as quorum-sensing, policing, mutual recognition, and population structure can also account for their prevalence. The picture that emerges from these considerations is therefore that the exuberance of bacterial group-level behavior does not always require highly individuated multicellular clusters.

Acknowledgments I thank Peter Godfrey-Smith, Georg Toepfer, Maureen O'Malley, and Pierrick Bourrat for invaluable feedback on earlier versions of this paper, as well as two anonymous referees for their extremely helpful comments. I also thank Hannah Read for her help and support.

\section{References}

Adams DG (2000) Cyanobacterial phylogeny and development: questions and challenges. In: Brun Y, Shimkets L (eds) Prokaryotic development. ASM Press, Washington, DC

Andrews JH (1998) Bacteria as modular organisms. Ann Rev Microbiol 52(1):105-126

Boyle R, Lenton T (2006) Fluctuation in the physical environment as a mechanism for reinforcing evolutionary transitions. J Theor Biol 242(4):832-843

Branda SS, Kolter R (2004) Multicellularity and biofilms. In: Ghannoum MA, O’Toole GA (eds) Microbial biofilms. ASM Press, Washington, DC, pp 20-29

Burkholder WF, Grossman AD (2000) Regulation of the initiation of endospore formation in Bacillus subtilis. In: Brun Y, Shimkets L (eds) Prokaryotic development. ASM Press, Washington, DC, pp 151-166

Burnham JC, Collart SA, Highison BW (1981) Entrapment and lysis of the cyanobacterium Phormidium luridum by aqueous colonies of Myxococcus xanthus PCO2. Arch Microbiol 129(4):285-294

Buss LW (1987) The evolution of individuality. Princeton University Press, Princeton

Cao P, Dey A, Vassallo C, Wall D (2015) How myxobacteria cooperate. J Mol Biol 427(23):3709-3721

Claessen D, Rozen DE, Kuipers OP, Søgaard-Andersen L, Van Wezel GP (2014) Bacterial solutions to multicellularity: a tale of biofilms, filaments and fruiting bodies. Nat Rev Microbiol 12(2):115-124

Clarke E (2016) Levels of selection in biofilms: multispecies biofilms are not evolutionary individuals. Biol Philos 31:191-212

Crespi B (2001) The evolution of social behavior in microorganisms. Trends Ecol Evolut 16(4):178-183

Davies DG (2000) Physiological events in biofilm formation. In: Allison DG, Gilbert P, Lappin-Scott HM, Wilson M (eds) Community structure and co-operation in biofilms. Cambridge University Press, Cambridge, pp 37-52

de Queiroz K (1998) The general lineage concept of species, species criteria, and the process of speciation: a conceptual unification and terminological recommendations. In: Berlocher SH, Howard DJ (eds) Endless forms: species and speciation. Oxford University Press, New York, pp 57-75

de Queiroz K (2007) Species concepts and species delimitation. Syst Biol 56(6):879-886

Dragoš A, Lakshmanan N, Martin M, Horváth B, Maróti G et al (2017) Evolution of exploitative interactions during diversification in Bacillus subtilis biofilms. FEMS Microbiol Ecol 93(12):fix 155

Ereshefsky M, Pedroso M (2013) Biological individuality: the case of biofilms. Biol Philos 28:331-349

Ereshefsky M, Pedroso M (2015) Rethinking evolutionary individuality. Proc Natl Acad Sci USA 112(33):10126-10132 
Flores E, Herrero A (2010) Compartmentalized function through cell differentiation in filamentous cyanobacteria. Nat Rev Microbiol $8(1): 39$

Godfrey-Smith P (2008) Varieties of population structure and the levels of selection. Br J Philos Sci 59(1):25-50

Godfrey-Smith P (2009) Darwinian populations and natural selection. Oxford University Press, New York

Godfrey-Smith P (2013) Darwinian individuals. In: Bouchard F, Huneman $\mathrm{P}$ (eds) From groups to individuals: evolution and emerging individuality. MIT Press, Cambridge, pp 17-36

Grosberg RK, Strathmann RR (1998) One cell, two cell, red cell, blue cell: the persistence of a unicellular stage in multicellular life histories. Trends Ecol Evolut 13(3):112-116

Huneman P (2014) Individuality as a theoretical scheme. II. About the weak individuality of organisms and ecosystems. Biol Theory 9(4):374-381

Huxley J (1912) The individual in the animal kingdom. Cambridge University Press, Cambridge

Jones S, Elliot M (2018) 'Exploring' the regulation of streptomyces growth and development. Curr Opin Microbiol 42:25-30

Kroos L, Piggot PJ, Moran CP Jr (2008) Bacillus subtilis sporulation and other multicellular behaviors. In: Whitworth DE (ed) Myxobacteria: multicellularity and differentiation. ASM Press, Washington, DC, p 363

Kumar K, Mella-Herrera R, Golden J (2010) Cyanobacterial heterocysts. Cold Spring Harb Perspect Biol 2(4):a000315

Lee SF, Li YH, Bowden GH (1996) Detachment of Streptococcus mutans biofilm cells by an endogenous enzymatic activity. Infect Immun 64(3):1035-1038

Lewontin RC (1970) The units of selection. Ann Rev Ecol Syst 1:1-18

Martí S, Rodríguez-Baño J, Catel-Ferreira M, Jouenne T, Vila J et al (2011) Biofilm formation at the solid-liquid and air-liquid interfaces by acinetobacter species. BMC Res Notes 4(1):5

Mendelson N, Morales D, Thwaites J (2002) The mechanisms responsible for 2-dimensional pattern formation in bacterial macrofiber populations grown on solid surfaces: fiber joining and the creation of exclusion zones. BMC Microbiol 2(1):1

Mendelson NH (1999) Bacillus subtilis macrofibres, colonies and bioconvection patterns use different strategies to achieve multicellular organization. Environ Microbiol 1(6):471-477

Mendelson NH, Salhi B, Li C (1997) Physical and genetic consequences of multicellularity in Bacillus subtilis. In: Shapiro JA, Dworkin M (eds) Bacteria as multicellular organisms. Oxford University Press, New York, pp 339-365

Munoz-Dorado J, Marcos-Torres F, Garcia-Bravo E, Moraleda-Munoz A, Perez J (2016) Myxobacteria: moving, killing, feeding, and surviving together. Front Microbiol 7(781):1-18

Nadell CD, Xavier JB, Foster KR (2009) The sociobiology of biofilms. FEMS Microbiol Rev 33(1):206-224

Okasha S (2005) Multi-level selection and the major transitions in evolution. Philos Sci 72(5):1013-1025

Paidhungat M, Setlow P (2002) Spore germination and outgrowth. In: Sonenshein AL, Losick R, Hoch J (eds) Bacillus subtilis and its relatives: from genes to cells. ASM Press, Washington, DC, pp 537-548

Papenfort K, Bassler B (2016) Quorum sensing signal-response systems in gram-negative bacteria. Nat Rev Microbiol 14(9):576

Pradeu T (2010) What is an organism? An immunological answer. Hist Philos Life Sci 32(2-3):247-267
Pradeu T (2016) Organisms or biological individuals? Combining physiological and evolutionary individuality. Biol Philos 31(6):797-817

Ratcliff WC, Denison RF, Borrello M, Travisano M (2012) Experimental evolution of multicellularity. Proc Natl Acad Sci USA 109(5):1595-1600

Roze D, Michod R (2001) Mutation, multilevel selection, and the evolution of propagule size during the origin of multicellularity. Am Natl 158(6):638-654

Schirrmeister BE, Antonelli A, Bagheri HC (2011) The origin of multicellularity in cyanobacteria. BMC Evolut Biol 11(1):45

Shapiro JA (1988) Bacteria as multicellular organisms. Sci Am 258(6):82-89

Shapiro JA (1997) Multicellularity: the rule, not the exception. In: Shapiro JA, Dworkin M (eds) Bacteria as multicellular organisms. Oxford University Press, New York, pp 14-49

Shimkets LJ (2000) Growth, sporulation, and other tough decisions. In: Shimkets LJ, Brun Y (eds) Prokaryotic development. ASM Press, Washington, DC, pp 277-284

Shimkets LJ, Dworkin M, Reichenbach H (2006) The myxobacteria. The prokaryotes: a handbook of the biology of bacteria. Springer, New York, pp 31-115

Skillings D (2016) Holobionts and the ecology of organisms: multispecies communities or integrated individuals? Biol Philos 31(6):875-892

Sonenshein AL (2000) Endospore-forming bacteria: an overview. In: Shimkets LJ, Brun Y (eds) Prokaryotic development. ASM Press, Washington, DC, pp 133-150

Steinberg N, Rosenberg G, Keren-Paz A, Kolodkin-Gal I (2018) Collective vortex-like movement of Bacillus subtilis facilitates the generation of floating biofilms. Front Microbiol 9:9550

Stoodley P, Sauer K, Davies DG, Costerton JW (2002) Biofilms as complex differentiated communities. Ann Rev Microbiol 56(1):187-209

Tecon R, Leveau J (2016) Symplasmata are a clonal, conditional, and reversible type of bacterial multicellularity. Sci Rep 6:31914

Travisano M, Velicer GJ (2004) Strategies of microbial cheater control. Trends Microbiol 12(2):72-78

Velicer GJ, Hillesland KL, Whitworth DE (2008) Why cooperate? The ecology and evolution of myxobacteria. In: Müller F, Jacobsen (eds) Myxobacteria: multicellularity and differentiation. ASM Press, Washington, DC, pp 17-40

Velicer GJ, Kroos L, Lenski RE (2000) Developmental cheating in the social bacterium Myxococcus xanthus. Nature 404(6778):598-601

Velicer GJ, Yu YN (2003) Evolution of novel cooperative swarming in the bacterium Myxococcus xanthus. Nature 425(6953):75-78

Vlamakis H, Chai Y, Beauregard P, Losick R, Kolter R (2013) Sticking together: building a biofilm the Bacillus subtilis way. Nat Rev Microbiol 11(3): 157

West SA, Griffin AS, Gardner A, Diggle SP (2006) Social evolution theory for microorganisms. Nat Rev Microbiol 4(8):597-607

Winstanley C, O'Brien S, Brockhurst M (2016) Pseudomonas aeruginosa evolutionary adaptation and diversification in cystic fibrosis chronic lung infections. Trends Microbiol 24(5):327-337

Wolk CP (2000) Heterocyst formation in Anabaena. Prokaryotic development. ASM Press, Washington, DC, pp 51-81 